\title{
Interferon-Gamma Impairs Maintenance and Alters Hematopoietic Support of Bone Marrow Mesenchymal Stromal Cells
}

\author{
Marieke Goedhart, ${ }^{1, *}$ Anne S. Cornelissen,, ${ }^{1, \star}$ Carlijn Kuijk, Sulima Geerman, \\ Marion Kleijer, Jaap D. van Buul, Stephan Huveneers, ${ }^{2,+}$ Marc H.G.P. Raaijmakers, \\ Howard A. Young, Monika C. Wolkers, ${ }^{4}$ Carlijn Voermans, ${ }^{1, \dagger}$ and Martijn A. Nolte ${ }^{1, \dagger}$
}

Bone marrow (BM) mesenchymal stromal cells (MSCs) provide microenvironmental support to hematopoietic stem and progenitor cells (HSPCs). Culture-expanded MSCs are interesting candidates for cellular therapies due to their immunosuppressive and regenerative potential which can be further enhanced by pretreatment with interferon-gamma (IFN- $\gamma$ ). However, it remains unknown whether IFN- $\gamma$ can also influence hematopoietic support by BM-MSCs. In this study, we elucidate the impact of IFN- $\gamma$ on the hematopoietic support of BM-MSCs. We found that IFN- $\gamma$ increases expression of interleukin (IL)- 6 and stem cell factor by human BM-MSCs. IFN- $\gamma-$ treated BM-MSCs drive HSPCs toward myeloid commitment in vitro, but impair subsequent differentiation of HSPC. Moreover, IFN- $\gamma$-ARE-Del mice with increased IFN- $\gamma$ production specifically lose their BM-MSCs, which correlates with a loss of hematopoietic stem cells' quiescence. Although IFN- $\gamma$ treatment enhances the immunomodulatory function of MSCs in a clinical setting, we conclude that IFN- $\gamma$ negatively affects maintenance of BM-MSCs and their hematopoietic support in vitro and in vivo.

Keywords: bone marrow, MSC, interferon-gamma, HSC, support

\section{Introduction}

$\mathrm{M}$ AINTENANCE OF HEMATOPOIETIC stem cells (HSCs) is key for life-long blood cell formation, which requires an appropriate balance between HSC quiescence, self-renewal, and differentiation. Therefore, HSCs reside in vascular niches in the bone marrow (BM) that consist of endothelial cells and perivascular mesenchymal stromal cells (MSCs)[1]. These cells promote HSC maintenance through secretion of hematopoietic support factors, such as stem cell factor (SCF) and CXCL12, which stimulate survival and attract HSCs to the niche, respectively. Conditional deletion of these factors from vascular niche cells results in a loss of quiescent HSCs and long-term repopulating activity $[2,3]$.
MSCs are present at very low frequencies in the BM and BM aspirates [4,5]. To obtain sufficient cell numbers for clinical applications, MSCs must be ex vivo expanded. Congruent to their role as supporting stromal cells in the vascular niche, expanded BM-MSCs also provide hematopoietic support to HSCs ex vivo [6]. MSCs also have the potential to generate other mesenchymal cell types, such as osteoblasts, chondrocytes, and adipocytes [5], indicating that they also may play a critical role in tissue regeneration. Indeed, cultureexpanded BM-MSCs can engraft in osteogenesis imperfecta patients and attenuate the disease by forming osteoblasts that stimulate dense bone formation [7]. Furthermore, cultureexpanded BM-MSCs exhibit immunoregulatory properties toward numerous innate and adaptive effector cells [8].

\footnotetext{
${ }^{1}$ Sanquin Research and Landsteiner Laboratory, Department of Hematopoiesis, Academic Medical Center, University of Amsterdam, Amsterdam, Netherlands.

${ }^{2}$ Sanquin Research and Landsteiner Laboratory, Department of Molecular Cell Biology, Academic Medical Center, University of Amsterdam, Amsterdam, Netherlands.

${ }^{3}$ Department of Hematology and Erasmus Stem Cell Institute, Erasmus MC Cancer Institute, Rotterdam, Netherlands.

${ }^{4}$ Cancer and Inflammation Program, Center for Cancer Research, National Cancer Institute, Frederick, Maryland.

$*$ These authors are co-first authors.

${ }^{\dagger}$ These authors are co-senior authors.

tCurrent affiliation: Department of Medical Biochemistry, Academic Medical Center, University of Amsterdam, Amsterdam, Netherlands.

(C) Marieke Goedhart et al., 2018; Published by Mary Ann Liebert, Inc. This Open Access article is distributed under the terms of the Creative Commons Attribution Noncommercial License (http://creativecommons.org/licenses/by-nc/4.0/), which permits any noncommercial use, distribution, and reproduction in any medium, provided the original author(s) and the source are cited.
} 
BM-MSCs are thus attractive candidates for cellular therapy after HSC transplantation to promote rapid hematopoietic recovery and reduce the incidence and severity of graftversus-host disease (GvHD) [9,10].

Expansion of MSCs ex vivo enables their modification with external factors to optimize them for cellular therapy. Recently, there has been considerable interest in the effect of cytokines on the therapeutic efficacy of culture-expanded BM-MSCs. Many studies support the notion that the immunosuppressive function of BM-MSCs is strongly enhanced by exposure to the proinflammatory cytokine interferon-gamma (IFN- $\gamma$ ) (reviewed in Ref. [11]). Stimulation of BM-MSCs with IFN- $\gamma$ before their administration in mouse models of acute GvHD is associated with reduced $\mathrm{T}$ cell proliferation and prolonged survival $[12,13]$. Other studies have suggested that preactivation with IFN- $\gamma$ enhances the migratory capacities and reparative properties of BM-MSCs [14,15], which is desirable, as MSCs have very limited migratory capacities (reviewed in Ref. $[16,17])$. Moreover, IFN- $\gamma$ stimulation before cryopreservation can enhance MSC fitness after thawing [18]. Thus, IFN- $\gamma$ can potentiate the immunomodulatory and tissue regenerative capacity of MSCs, and thereby generate a potent cell population for application in various clinical conditions (reviewed in Ref. [19]). To date, however, little attention has been devoted to the effect of IFN- $\gamma$ on hematopoietic support by BM-MSCs.

We and others have reported that IFN- $\gamma$ can have a detrimental effect on the maintenance and self-renewal of HSCs during inflammatory conditions [20-22], but the impact on BM-MSCs is unclear. One possibility is that MSCs are activated by IFN- $\gamma$ and thereby enhance their hematopoietic supportive capacity during inflammatory stress. Alternatively, a negative effect of IFN- $\gamma$ on the hematopoietic support function of MSCs can be one of the mechanisms underlying hematopoietic exhaustion observed in HSCs during inflammation [23]. Considering the essential survival and quiescence enforcing signals for HSCs provided by MSCs in vivo, it is important to determine whether the supportive function of MSCs is influenced by a proinflammatory environment. In addition, if IFN- $\gamma$ also enhances the ability of cultureexpanded BM-MSCs to support the hematopoietic function of HSCs, this may further expand the clinical conditions for which BM-MSCs could be applied as cellular therapy.

The objective of the present study is therefore to examine the impact of IFN- $\gamma$ on hematopoietic support by BM-MSCs. We demonstrate that, in contrast to other functional characteristics, the hematopoietic support function of BM-MSCs does not benefit from pretreatment with IFN- $\gamma$. In fact, we show that IFN- $\gamma$ impairs maintenance of BM-MSCs in vitro and in vivo, which has detrimental effects on HSC function.

\section{Materials and Methods}

\section{Human BM-MSC culture}

BM samples were obtained from patients (age range 18-70 years) undergoing median sternotomy for cardiac surgery, after informed consent and approval of the medical Ethics Review Board of the AMC (MEC:04/042\#04.17.370). Briefly, $\mathrm{BM}$ mononuclear cells were isolated by density gradient centrifugation (Ficoll-Paque) and seeded in T80 tissue culture flasks $\left(2.5-3.75 \times 10^{5}\right.$ cells $/ \mathrm{cm}^{2}$ ) in DMEM GlutaMAX (low glucose) supplemented with $10 \%$ fetal calf serum (FCS) and
$1 \%$ penicillin-streptomycin. After $48 \mathrm{~h}$, nonadherent cells were removed. When reaching $80 \%-90 \%$ confluency (3-4 weeks for each passage), MSCs were trypsinized with TrypLE (Gibco) and further cultured. For the experiments, MSCs up until passage 2 were used.

\section{IFN- $\gamma$ stimulation}

IFN- $\gamma$ stimulation of MSC was initiated 2 weeks after the initial isolation. In brief, normal culture medium was removed and culture medium with $10 \mathrm{ng} / \mathrm{mL}$ recombinant human IFN- $\gamma$ (Imukin, Boehringer Ingelheim), was added. Culture medium of IFN- $\gamma$-stimulated MSC, now referred to as MSC- $\gamma$, was refreshed once a week and experiments were initiated 3-4 days after a medium change.

\section{Human BM-MSC and hematopoietic stem and progenitor cell coculture}

Cocultures of MSCs and hematopoietic stem and progenitor cells (HSPCs) were performed as previously described [24]; graphical summary of the experimental set-up is shown in Supplementary Fig. S1A (Supplementary Data are available online at www.liebertpub.com/scd). In brief, MSC and MSC- $\gamma$ were harvested, IFN- $\gamma$ was washed away and cells were subsequently seeded in 48-well plates at the density of 40,000 cells/well (equal cell numbers for MSC and MSC- $\gamma$ ). Cord blood (CB) was collected according to the guidelines of Eurocord Nederland and CD $34^{+}$cells were isolated as previously described [25]. Ten thousand CD34 ${ }^{+}$ cells were added onto the stromal layers and cocultured in MyeloCult (H5100; Stem Cell Technologies) for 7 days at $37^{\circ} \mathrm{C}$ and $5 \% \mathrm{CO}_{2}$. During the coculture, no IFN- $\gamma$ was present. On day 7 , the complete coculture was trypsinized with TrypLE (Gibco) and harvested. Total hematopoietic cells were characterized for surface expression of various markers using an LSRII flow cytometer (BD Biosciences) and cells were counted with Cyto-Cal fluorescent beads (Thermo Scientific, Fremont, CA). The gating strategy for the surface marker expression analysis is depicted in Supplementary Fig. S1B. MSCs, present in the coculture, were excluded from the hematopoietic surface marker expression on the flow cytometer based on CD73 expression. On average, 5,000 gated events (HSPC) were acquired per sample. Cell counting was performed using Cyto-Cal fluorescent beads $\left(1 \times 10^{6} / \mathrm{mL}\right)$ that were added to the cell suspension. Cell concentration was calculated with the following formula: $(\mathrm{A} / \mathrm{B}) \times(\mathrm{C} / \mathrm{D})$ in which $\mathrm{A}=$ number of recorded cell events, $\mathrm{B}=$ number of recorded bead events, $\mathrm{C}=$ assigned bead count (beads $/ 50 \mu \mathrm{L}$ ), and $\mathrm{D}=$ volume of sample $(\mu \mathrm{L})$.

\section{Human hematopoietic colony formation}

For hematopoietic colony formation assays, the nonadherent fraction of hematopoietic cells was harvested after 7 days of coculturing with MSCs and a volume of $500 \mu \mathrm{L}$ was plated in MethoCult (H4434; Stem Cell Technologies) in duplicates in 24-well plates. Cultures were scored for the presence of hematopoietic colonies (CFU-granulocyte, erythrocyte, monocyte, megakaryocyte [GEMM], colonyforming unit granulocyte-monocyte [CFU-GM] or burstforming unit-erythroid [BFU-E]) in duplo after 14 days of incubation at $37^{\circ} \mathrm{C}$ and $5 \% \mathrm{CO}_{2}$. 


\section{Mice}

For murine experiments, IFN- $\gamma$ AU-rich elements (ARE)Del mice and wild-type (WT) mice were used [26]. Mice were maintained on a C57BL/6 background in the animal facilities of the Netherlands Cancer Institute (Amsterdam, The Netherlands) in specific pathogen-free conditions. Mice were given standard chow and acidified drinking water ad libitum. Femurs and tibiae of Nestin-GFP mice [27] were used for confirmation of our murine MSC gating strategy. All animal experiments were approved by the Experimental Animal Committee of the Netherlands Cancer Institute, according to institutional and national guidelines.

\section{Murine BM cell isolation}

Murine BM cells were obtained by mildly crushing femurs and tibiae with a mortar and pestle. For stromal cell isolations, bones were digested with Liberase TM grade and DNAse I (Roche Diagnostics) for $30 \mathrm{~min}$ at $37^{\circ} \mathrm{C}$. All singlecell suspensions were filtered through a $70-\mu \mathrm{m}$ cell strainer.

\section{Murine long-term-HSC cultures}

For 7-day HSC cultures, 500 HSCs $\left(\mathrm{Lin}^{-} \mathrm{c}-\mathrm{Kit}^{-}\right.$ Sca- $1^{+} \mathrm{CD} 48^{-} \mathrm{CD} 150^{+}$) were sorted and cultured in 96-well plates in X-VIVO 15 medium (Lonza) with $2 \mathrm{ng} / \mathrm{mL}$ thrombopoietin and SCF and $5 \mathrm{ng} / \mathrm{mL}$ IL-3, IL-6, and Flt3L. Cells were cultured at $37^{\circ} \mathrm{C}$ in a humidified incubator at $5 \% \mathrm{CO}_{2}$. All cytokines were obtained from PeproTech.

\section{Flow cytometry and cell sorting}

FACSCanto II (BD Biosciences), LSR II (BD Biosciences), and FACSAria (BD Biosciences) were used for flow cytometric analysis and cell sorting. Human MSCs were stained with Near IR Live-Dead marker (Life Technologies), CD45 (clone HI30), CD34 (clone 8G12), CD73 (clone AD2), CD90 (clone 5E10), CD105 (clone 266), human leukocyte antigen ABC (HLA-ABC) (clone G46-2.6), and human leukocyte antigen DR (HLA-DR) (clone G46-6; all from BD Biosciences). On average, the purity of cells that fulfill the MSC criteria $\left(\mathrm{CD} 45^{-}, \mathrm{CD} 34^{-}, \mathrm{CD} 73^{+}, \mathrm{CD} 90^{+}\right.$, and $\mathrm{CD} 105^{+}$) was $96 \%$. Human HSPCs were stained with Near IR Live-Dead marker (Life Technologies), CD13 (clone WM15), CD14 (clone M5E2), CD33 (clone WM53) CD34 (clone 8G12), CD45 (clone HI30), CD235a (clone GA-R2; all from BD biosciences), CD36 (clone M1613; Sanquin), and CD41 (clone 5B12; Dako). For flow cytometric analysis and cell sorting of murine MSC, whole digested BM was enriched for stromal cells by negative depletion of hematopoietic cells using CD45 microbeads (Miltenyi Biotec), and cells were stained for CD45.2 (clone 104), Ter119 (clone TER-119), CD31 (clone 390), PDGFR $\alpha$ (clone APA5), andCD51 (clone RMV-7; all from eBioscience). For flow cytometric analysis of murine HSC, whole BM was stained for lineage markers, (CD4 [clone GK1.5], CD8 [clone 53-6.7], B220 [clone RA36B2], CD11b [clone M1/70], Gr-1 [clone RB6-8C5], and Ter119 [clone TER-119]); Sca-1 (clone D7), c-kit (clone 2B8), CD48 (clone HM48-1), and CD150 (clone TC1512F12.2; all from eBioscience). Intracellular staining of Ki-67 or isotype control (BD Biosciences) was performed after fixation with the Foxp3/Transcription Factor Staining buffer set (eBioscience). For intracellular staining of IFN- $\gamma$ in $\mathrm{T}$ cell and NK cell subsets, whole BM was stained with biotinlabeled antibodies against B220 (clone RA3-6B2), CD11b (clone M1/70), Gr-1 (clone RB6-8C5), and Ter119 (clone TER-119; all from eBioscience), and enriched for T cells NK cells by negative depletion of biotin-labeled cells using streptavidin microbeads (Miltenyi Biotec). Subsequently, the enriched cell suspension was cultured for $4 \mathrm{~h}$ in IMDM (Lonza) supplemented with $10 \%$ FCS and 0.1\% 2-Mercaptoethanol (Sigma) in the presence of Brefeldin A (eBioscience). Subsets of T cells, NK cells, and NK T cells were identified with antibodies against CD3 (clone 17A2), CD4 (clone GK1.5), CD8 (clone 53-6.7), CD44 (clone IM7), CD62L (clone MEL-14), and NK1.1 (clone PK136; all from eBioscience). Intracellular staining for IFN- $\gamma$ protein was performed after fixation with the Cytofix/Cytoperm Solution Kit (BD Biosciences). Before sorting murine long-term HSC (LT-HSC), whole BM was stained with the biotinylated lineage markers and enriched for progenitors by negative depletion of lineage cells using streptavidin microbeads (Miltenyi Biotec).

\section{Quantitative real-time polymerase chain reaction}

For human samples, total RNA was extracted with the RNeasy Mini Kit according to the manufacturer's instructions (Qiagen). First-strand cDNA synthesis was performed on the full volume of RNA solution, without measuring the quantity of RNA, using M-MLV reverse transcriptase and random hexamer primers (both from Invitrogen). For murine samples, RNA was extracted using TRIzol (Invitrogen) and complementary DNA was made with iScript Reverse Transcription Supermix for quantitative real-time polymerase chain reaction reactions (RT-qPCR; Bio-Rad). RTqPCRs were performed on a StepOnePlus (Applied Biosystems) using TaqMan or SYBR Green dye for detection (both from Applied Biosystems). Forty cycles were used for amplification. Data were normalized using ABL1 (human) and Cyclophilin (murine) as reference genes using the double delta $\mathrm{Ct}$ method: $2^{-(\Delta \mathrm{Ct} \text { sample }-\Delta \mathrm{Ct} \text { reference gene) }}$. Primer sequences and amplicon sizes are listed in Table 1.

\section{Statistics}

Statistical analyses were performed with GraphPad Prism 7. Mean values plus or minus standard deviation or standard error of the mean are shown. $* P<0.05 ; * * P<0.01 ; * * * P<0.001$.

\section{Results}

IFN- $\gamma$ exposure alters the expression of hematopoietic cytokines secreted by MSCs

We first determined the impact of IFN- $\gamma$ on the phenotype and function of human MSC. MSCs were continuously exposed to IFN- $\gamma$ in culture for 1-2 passages. Culturing in the presence of IFN- $\gamma$ resulted in a reduced expansion of MSC (Supplementary Fig. S2a). As expected for cells exposed to IFN- $\gamma$ [28], HLA-ABC, and HLA-DR expression was increased (Fig. 1a). Moreover, phenotypic expression of the classical MSC markers ( $>95 \%$ according to the ISCT criteria [29]) CD73, CD90, and CD105 remained stable during the course of the experiment. This is important as others have shown that IFN- $\gamma$ can alter multilineage differentiation of MSCs with a skewing toward osteogenesis and a reduction in adipogenesis [30,31]. 
Table 1. Primer Sequences

Gene

Human $A B L 1$ (forward)

Human $A B L 1$ (reverse)

Human $A B L 1$ (probe)

Human CXCL12 (forward)

Human CXCL12 (reverse)

Human IL6 (forward)

Human IL6 (reverse)

Human IDOI (forward)

Human IDOI (reverse)

Human SCF (forward)

Human $S C F$ (reverse)

Mouse $\mathrm{Cxcl12}$ (forward)

Mouse $\mathrm{Cxcl12}$ (reverse)

Mouse $S c f$ (forward)

Mouse $S c f$ (reverse)

Mouse Nes (forward)

Mouse Nes (reverse)

Mouse Ppia (forward)

Mouse Ppia (reverse)
Primer sequence

Amplicon size (bp)

$5^{\prime}$ TGG AGA TAA CAC TCT AAG CAT AAC TAA AGG T $3^{\prime}$

5' GAT GTA GTT GCT TGG GAC CCA 3'

5' CCA TTT TTG GTT TGG GCT TCA CAC CAT T $3^{\prime}$

$5^{\prime}$ TCT CAA AAT TCT CAA CAC TCC AAA CT $3^{\prime}$

5' GCA CAC TTG TCT GTT GTT GTT CTT C 3'

5' TCT CCA CAA GCG CCT TCG 3'

5' CTC AGG GCT GAG ATG CCG 3'

$5^{\prime}$ ACC ATA TTG ATG AAG AAG TGG GC 3'

$5^{\prime}$ TGA ACA TCC AGT CAT TAT AAA AAT CAG G 3'

$5^{\prime}$ AGC GCT GCC TTT CCT TAT GA 3'

5' GA CGA GAG GAT TAA ATA GGA GCA 3'

5' CAG AGC CAA CGT CAA GCA TCT 3'

5' GGT CAA TGC ACA CTT GTC TGT TGT 3'

5' AAG GAG ATC TGC GGG AAT CC 3'

5' CCA TCC CGG CGA CAT AGT T 3'

5' GCT GGA ACA GAG ATT GGA AGG 3'

5' CCA GGA TCT GAG CGA TCT GAC 3'

$5^{\prime}$ ACC CAT CAA ACC ATT CCT TCT GTA $3^{\prime}$

$5^{\prime}$ TGA GGA AAA TAT GGA ACC CAA AGA 3'
124

76

193

81

85

101

109

125

112

Primer sequences and amplicon sizes for the human and murine genes analyzed by RT-qPCR.

SCF, stem cell factor; RT-qPCR, quantitative real-time polymerase chain reaction.

MSCs produce a plethora of hematopoietic supportive factors, including IL-6, SCF, and CXCL12, and we examined whether IFN- $\gamma$ altered the expression of these supporting mediators by MSC. As a positive control, we could demonstrate that IFN- $\gamma$ increased the expression of indoleamine 2,3 dioxygenase, a direct target gene of IFN- $\gamma$ [32]. Interestingly, while the levels of $C X C L 12$ mRNA were unaffected by IFN- $\gamma$ exposure, we observed a significant increase in IL6 mRNA expression, a factor that activates myelopoiesis in response to infection and chronic inflammation [33-35]. Furthermore, the expression of $S C F$, which is involved in HSC maintenance [2], was also increased (Fig. 1b). Altogether, these data show that IFN- $\gamma$ exposure enhances expression of hematopoietic cytokines, while stably maintaining the expression of classical MSC markers and CXCL12.

\section{IFN- $\gamma$ exposure alters the hematopoietic support function of MSCS}

To examine the impact of IFN- $\gamma$ on the hematopoietic support function of MSCs, we used an in vitro coculture system of human BM-MSCs and umbilical CB CD34 HSPCs, in which the MSCs strongly support both the maintenance and the outgrowth of HSPCs [24,36,37]. Viable MSCs, expanded without or with IFN- $\gamma$ (MSC vs. MSC- $\gamma$ ), were cocultured with $\mathrm{CB}$ CD $34^{+}$HSPCs for 7 days (Experimental set-up shown in Supplementary Fig. S1a; representative images in Supplementary Fig. S2b). After the coculture, all cells were harvested and total hematopoietic cells were counted. To validate that the effects of IFN- $\gamma$ stimulation of MSCs lasts for 7 days, MSCs were phenotypically analyzed before and after the coculture. Upregulation of HLA-ABC and HLA-DR was still present at the end of the coculture, suggesting that the effect of IFN- $\gamma$ stimulation is maintained during the coculture (Supplementary Fig. S3).

In contrast to the increase in hematopoietic cytokine production, we observed no significant differences in total hematopoietic cell counts between MSC and MSC- $\gamma$ conditions
(Fig. 1c). Similar results were obtained when MSCs were cultured with IFN- $\gamma$ for $40-48 \mathrm{~h}$, a timeframe that has been reported to enhance immunomodulatory, migratory, and regenerative capacities of MSCs for clinical applications (Supplementary Fig. S4a) $[11,14,15]$. This suggests that both long- and short-term IFN- $\gamma$ stimulation of MSCs do not lead to a gain of function in hematopoietic support. To study whether MSC- $\gamma$ could boost the clonogenic capacity of HSPCs, we performed hematopoietic colony assays on cells derived from a 1-week coculture of HSPCs and MSCs. After 2 weeks, CFU-GM, BFU-E, and GEMM colonies were counted. No significant differences in progenitor supporting capacity were observed between MSC and MSC- $\gamma$, neither in the number nor the type of colonies (Fig. 1d). Similar results were obtained when MSCs were stimulated with IFN- $\gamma$ only for 40-48 h (Supplementary Fig. S4b). These data indicate that MSC- $\gamma$ could still support the maintenance of functional hematopoietic progenitor cells. This was in accordance with flow cytometric analysis on HSPCs after 7 days of coculturing, as we found that the number of myeloid progenitor cells $\left(\mathrm{CD} 34^{+} / \mathrm{CD} 13 / 33^{+}\right.$cells) was maintained and even slightly increased $(P=0.0439)$ (Fig. 1e). However, the number of differentiated myeloid cells $\left(\mathrm{CD} 14^{+}\right.$and $\mathrm{CD}^{+} 6^{+}$cells) was strongly reduced when HSPCs were grown on MSC- $\gamma$ $(P=0.0014$ and 0.0067 , respectively) (Fig. 1e). This indicates that IFN- $\gamma$ treatment of MSCs supports myeloid commitment of HSPCs, but impairs subsequent maturation toward monocytes/macrophages. Hematopoietic cell viability after the coculture was similar between MSC and MSC- $\gamma$ conditions (data not shown). Moreover, CD41 (megakaryocyte maker) and CD235a (red cell lineage marker) expression on HSPC was not altered (Supplementary Fig. S5), suggesting that there is no lineage skewing upon coculturing with MSC- $\gamma$.

\section{BM-MSCs are reduced in vivo after IFN- $\gamma$ exposure}

These data demonstrate that IFN- $\gamma$ does not boost the hematopoietic support function of in vitro-expanded MSCs, 


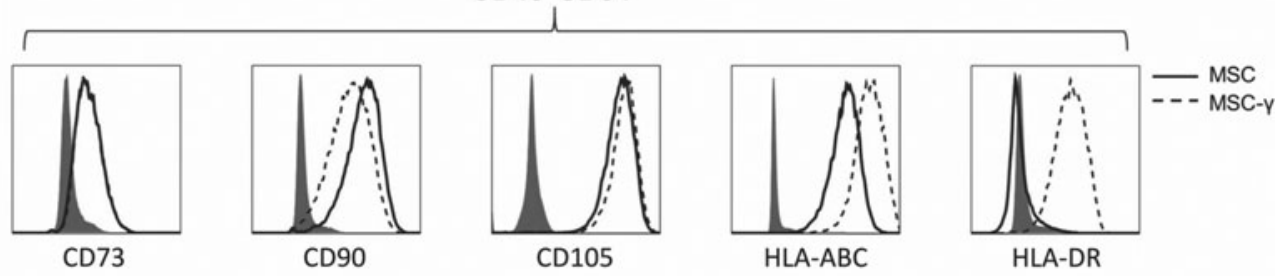

B
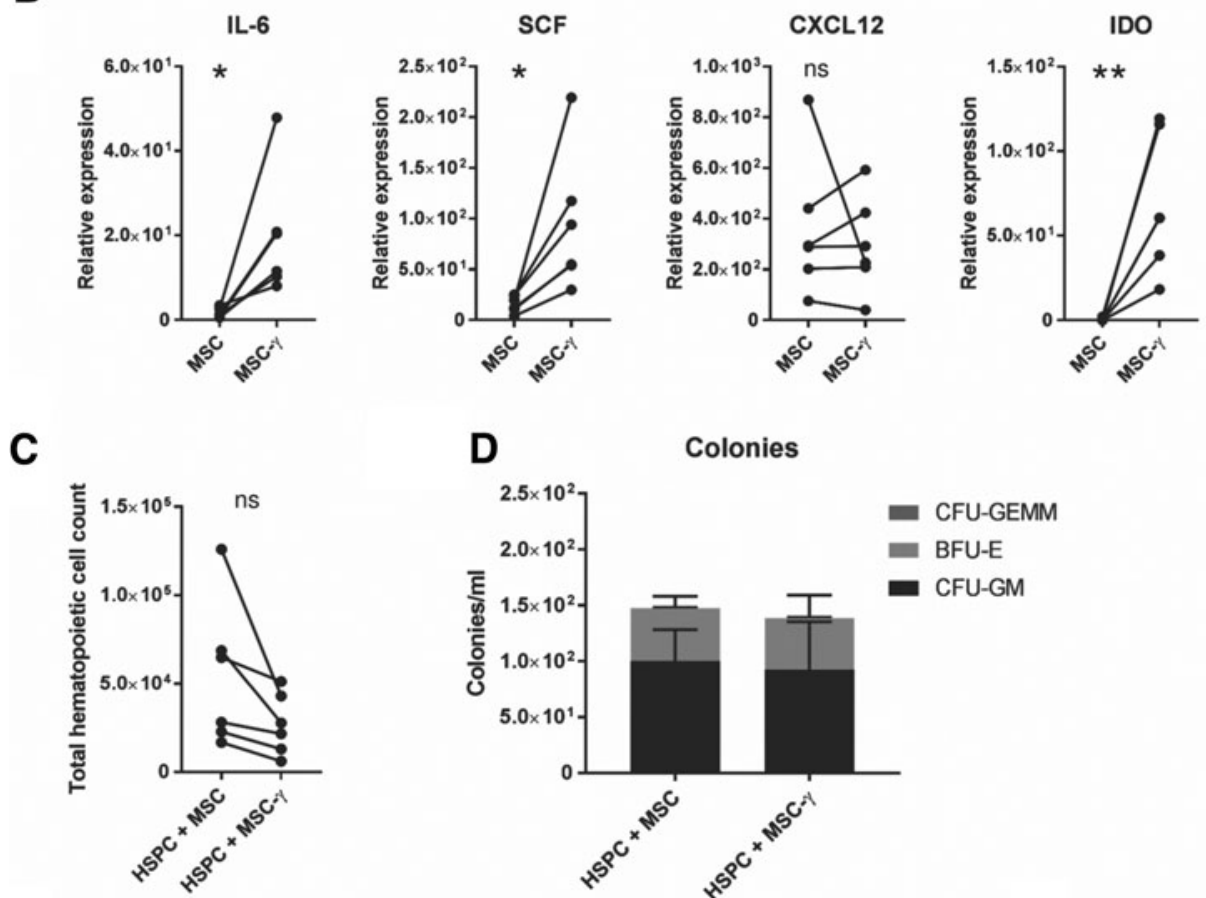

D

Colonies
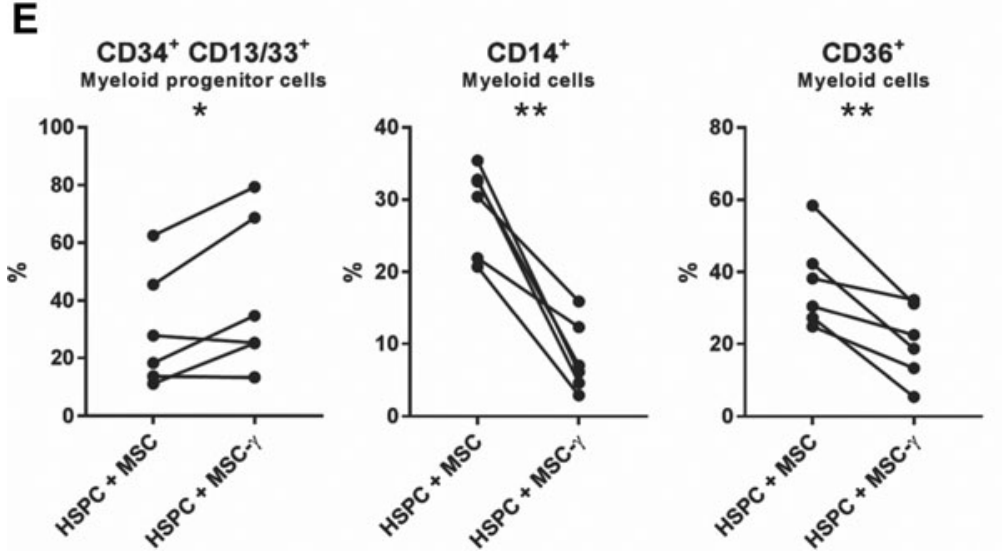

$\mathbf{F}$

MSC expansion

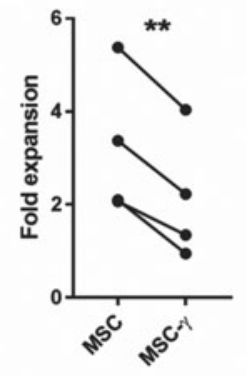

FIG. 1. IFN- $\gamma$ alters hematopoietic support by MSCs. (A) Phenotypical flow cytometric analysis of unstimulated (MSC) or IFN- $\gamma$-stimulated MSCs (MSC- $\gamma$ ). MSCs were negative for CD34 and CD45 and positive for CD73, CD90, and CD105. Upon IFN- $\gamma$ stimulation HLA-ABC and HLA-DR were upregulated. Filled histograms represent isotype control. Flow cytometric analyses were performed for all MSC donors used in the coculture experiments $(n=6)$. Representative example. (B) mRNA expression of $I L-6, S C F, C X C L 12$, and $I D O$ in MSC and MSC- $\gamma$ was analyzed by QPCR. ABLI was used as a housekeeping gene to normalize and determine the expression levels $(n=5-6)$. (C) Total hematopoietic cell counts (absolute) after 7 days of coculturing MSC or MSC- $\gamma$ with HSPCs $(n=6)$. (D) After 7 days of coculturing MSC or MSC $-\gamma$ with HSPCs, a volume of $500 \mu \mathrm{L}$ was seeded in methylcellulose medium to examine the total supporting capacity of the culture. Colony-forming precursors were counted after 14 days of culturing. Bars represent the average total (mean-SD; $n=6$ ). (E) Flow cytometric analysis of hematopoietic cells after 7 days of coculturing MSC or MSC- $\gamma$ with HSPCs. Presence of CD $34^{+}-\mathrm{CD} 13 / 33^{+}$(myeloid progenitors), $\mathrm{CD}^{+} 4^{+}$, and CD36 (monocyte) hematopoietic cells was determined $(n=6)$. (F). Expansion of MSC and MSC- $\gamma$ cultured in M199 media supplemented with $10 \% \mathrm{FCS}, 1 \%$ penicillin-streptomycin, $20 \mu \mathrm{g} / \mathrm{mL}$ ECGF, and $8 \mathrm{IU} / \mathrm{mL}$ heparin (mean-SD; $n=5$; paired $t$-test). In all experiments MSC- $\gamma$ were continuously cultured in medium containing $10 \mathrm{ng} / \mathrm{mL}$ recombinant human IFN- $\gamma$. For each $n$-number, a unique MSC donor and a unique cord blood donor were used. $* P<0.05$; $* *<0.01$ paired $t$-test. CFU, colony-forming unit; GM, granulocyte-monocyte; GEMM, granulocyte-erythrocyte-monocyte-megakaryocyte; BFU-E, burst-forming unit-erythroid; MSC, mesenchymal stromal cell; HSPC, hematopoietic stem and progenitor cell; IFN- $\gamma$, interferon-gamma; SCF, stem cell factor; SD, standard deviation; IDO, indoleamine 2,3 dioxygenase; HLA-ABC, human leukocyte antigen ABC; HLA-DR, human leukocyte antigen DR; QPCR, quantitative real-time PCR; FCS, fetal calf serum; ECGF, endothelial cell growth factor. 
which is important for the exploration of the clinical potential and applicability of IFN- $\gamma$-stimulated MSCs. However, it could be that the in vivo impact of IFN- $\gamma$ on the HSC-supporting function of MSCs in the BM niches is quite different. As the biology and function of IFN- $\gamma$ is evolutionarily highly conserved between man and mouse, we addressed this question in a mouse model, in which IFN- $\gamma$ expression is moderately increased, without inducing an infection. We made use of a transgenic mouse model with a 162 nt deletion of the ARE in the $3^{\prime}$ untranslated region of the IFN- $\gamma$ gene. This deletion drives continuous IFN- $\gamma$ production, as evidenced by chronic low levels of IFN- $\gamma$ in serum $(10-30 \mathrm{pg} / \mathrm{mL})$ [26]. IFN- $\gamma$ production in the $\mathrm{BM}$ was also increased, as evidenced by a higher percentage of IFN- $\gamma$-producing NK cells, NKT cells, and $\mathrm{T}$ cell subsets compared in ARE-Del mice compared with WT controls (Fig. 2a, b). Importantly, chronic exposure to IFN- $\gamma$ did not alter total BM cell numbers in ARE-Del compared with WT controls (Fig. 2c).

Murine BM-MSCs were defined as CD45 Ter119- CD31 PDGFRa $^{+} \mathrm{CD}^{-} 1^{+}$cells (Fig. 2d) [38]. We confirmed that these cells produce the vast majority of the hematopoietic support factors CXCL12 and SCF in the BM, and that they are positive for the commonly used MSC-marker Nestin (Supplementary Fig. S6a, b) [38,39]. When assessing the impact of chronic in vivo exposure of IFN- $\gamma$ on BM stromal cells, we found that particularly BM-MSCs were significantly reduced in the BM stromal compartment of ARE-Del mice (Fig. 2e; Supplementary Fig. S7a), whereas other stromal subsets, including endothelial cells (Supplementary Fig. S7b), were not significantly affected.

When analyzing the functional characteristics of the remaining BM-MSCs of ARE-Del mice, we found that increased exposure to IFN- $\gamma$ did not affect the expression of CXCL12 and SCF on a per cell basis (Fig. 2f), suggesting that the persisting MSCs were still functionally intact. Importantly, analysis of young, 3-week-old mice, which still lack IFN- $\gamma$-producing cells in the BM (data not shown), revealed that MSCs do develop normally in ARE-Del mice, although rapidly decline when the mice age and IFN- $\gamma$ producing cells accumulate in the BM (Fig. 2g). In conclusion, using this sterile inflammation model, we demonstrate that increased IFN- $\gamma$ production induces loss of BMMSCs in vivo. This discovery is in line with previous studies $[32,40]$, as well as our own observations (Fig. 1f; Supplementary Fig. S2a) that IFN- $\gamma$ has a detrimental effect on the proliferative capacity of cultured human MSCs.

\section{IFN- $\gamma$-mediated loss of BM-MSCs coincides with loss of HSC quiescence}

As MSCs contribute to the maintenance and quiescence of HSCs $[3,39,41]$, we examined to what extent the IFN- $\gamma$ mediated loss of MSCs affected the composition of the hematopoietic progenitor compartment in ARE-Del mice. We identified HSPCs by classifying the HSC-containing Lineage $^{-}$Sca- $^{+}$c-kit $^{+}$(LSK) population and further subdividing HSCs and progenitors using the SLAM family receptors CD150 and CD48 (Fig. 3a) [42]. Within the LSK population of ARE-Del mice, we observed a skewed distribution of CD150 ${ }^{+} \mathrm{CD} 48^{-}$LT-HSC, CD $150^{+} \mathrm{CD} 48^{+}$short-term HSC (ST-HSC), and CD150-CD $48^{+}$multipotent progenitors (MPP): LT-HSCs were significantly underrepresented in the
LSK fraction of ARE-Del mice compared with WT controls, but this did not result in a significant decline of total LT-HSC numbers (Fig. 3b, c). However, Ki-67 staining revealed that LT-HSCs in ARE-Del mice exhibit a loss of quiescence: only $35 \%$ of LT-HSC in ARE-Del mice were quiescent compared with $70 \%$ in WT controls (Fig. 3d, e). Loss of quiescence in LT-HSC did not lead to increased self-renewal, as we did not observe increased numbers of LT-HSC in ARE-Del mice. Instead, it is more likely that loss of quiescence in LT-HSCs enhanced their subsequent differentiation toward more committed progenitors, which was corroborated by higher absolute numbers of ST-HSC and MPP in ARE-Del mice compared with WT controls (Fig. 3c).

To further investigate the functional capacities of ARE-Del LT-HSC, we sorted LT-HSC from WT and ARE-Del mice and performed an in vitro HSC culture assay. We previously showed that phenotypical LT-HSCs remain functionally intact in this assay and that they are capable of long-term, multilineage reconstitution [22]. We observed after 7 days that the absolute number of phenotypical LT-HSC was significantly decreased in ARE-Del compared with WT cultures (Fig. 3f). This loss of LT-HSC maintenance coincided with increased differentiation toward MPPs, myeloid progenitors, and differentiated myeloid cells, indicating that LT-HSC in ARE-Del mice lost their ability to self-renew and rapidly differentiated toward the myeloid lineage. We thus found decreased quiescence in LT-HSC from adult ARE-Del mice, which resulted in decreased maintenance of phenotypical LTHSC upon in vitro culture.

Finally, we anticipated that the LT-HSC pool would become exhausted upon aging and that the LT-HSCs could not be maintained in old ARE-Del mice. Indeed, when examining the BM of 1-year-old mice, we found that the number of BM-MSCs was still significantly reduced in ARE-Del compared with WT control mice (Fig. 3g), which coincided with a significant reduction in the absolute number of LTHSCs (Fig. 3h). These findings indicate that IFN- $\gamma$-mediated loss of BM-MSCs coincides with a decrease in HSC quiescence and a subsequent exhaustion of the LT-HSC pool upon aging. Thus, we conclude from these data that in vivo exposure of BM-MSC to IFN- $\gamma$ has a detrimental effect on MSC maintenance and is therefore destructive for long-term support of quiescent HSCs.

\section{Discussion}

IFN- $\gamma$ potentiates the immunomodulatory and tissue regenerative capacity of MSCs, and thereby generates a potent MSC population for application in various clinical conditions (reviewed in Ref. [19]). However, the effect of IFN- $\gamma$ on hematopoietic support of MSCs has thus far not been properly addressed. We show here that IFN- $\gamma$ impaired the ex vivo maintenance of human BM-MSCs, and did not further enhance their hematopoietic support capacity, but rather inhibited myeloid differentiation. The lack of increased hematopoietic support by MSC- $\gamma$ in our in vitro culture assays, despite their increased expression of SCF, could be due to the fact that cultured BM-MSC may already produce sufficient amounts of SCF for HSPC maintenance, with no additional effect of increased levels of SCF. In addition, the myeloid skewing that is expected upon upregulation of IL-6 [34] may be counteracted by potential other 

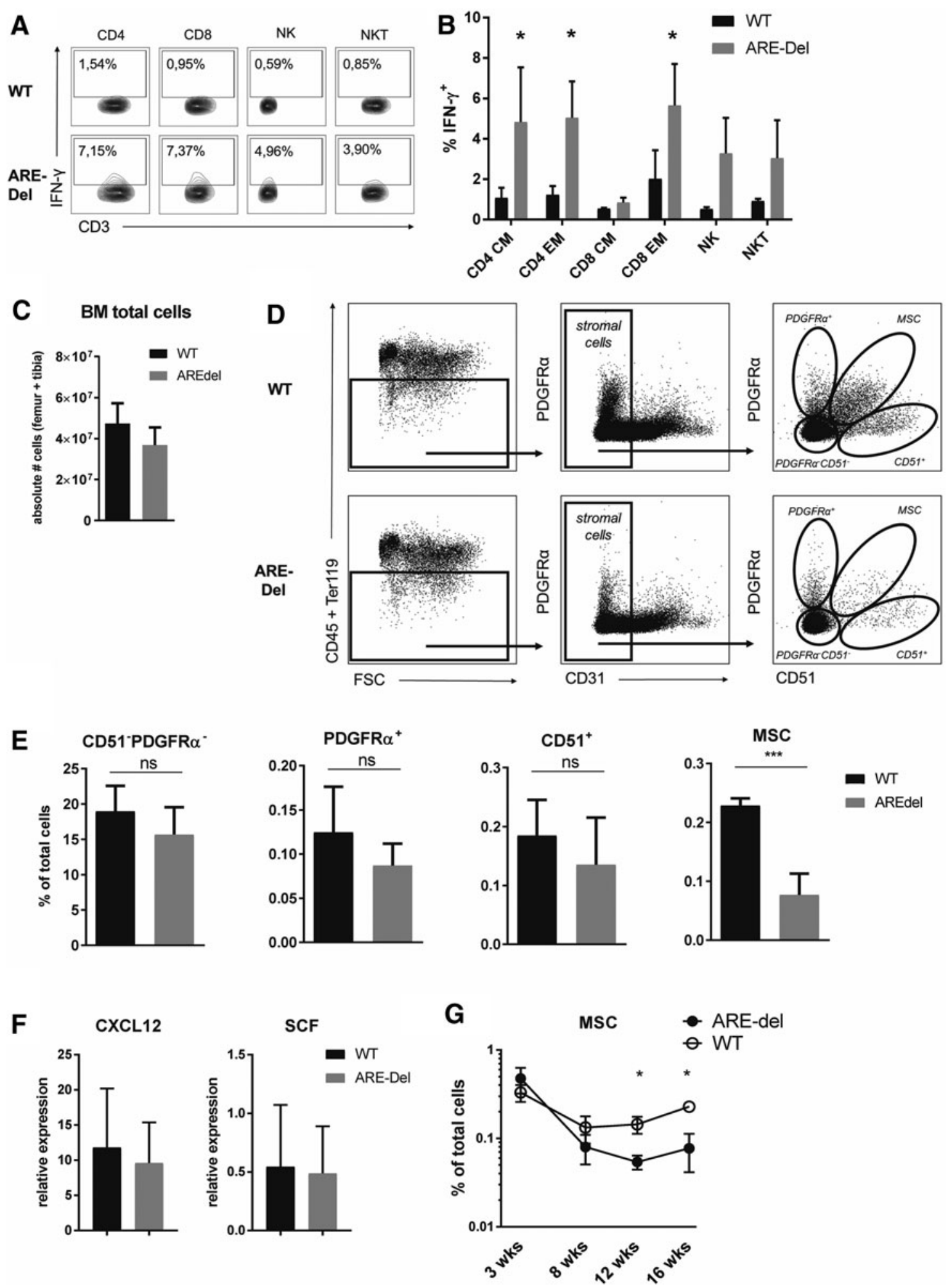

FIG. 2. BM-MSCs are reduced in vivo after IFN- $\gamma$ exposure. (A) Flow cytometric analysis and (B) quantification of IFN- $\gamma$ production by BM T cells and NK cells from ARE-Del mice and WT controls. CD4+ and CD8+ T cells were defined as $\mathrm{CD}^{+} \mathrm{CD}^{+} \mathrm{CD}^{-}$and $\mathrm{CD}^{+} \mathrm{CD}^{-} \mathrm{CD}^{+}$, respectively. $\mathrm{CM}=$ central memory $\left(\mathrm{CD} 44^{+} \mathrm{CD} 62 \mathrm{~L}^{+}\right)$. $\mathrm{EM}=\mathrm{effector}$ memory $\left(\mathrm{CD} 44^{+} \mathrm{CD} 62 \mathrm{~L}^{-}\right)$. NK cells were defined as CD3 ${ }^{-} \mathrm{CD}^{+} 6^{+}$and $\mathrm{NKT}$ cells were $\mathrm{CD} 3{ }^{+} \mathrm{CD} 56^{+}$. Cells were incubated for $4 \mathrm{~h}$ without any stimulus in the presence of Brefeldin A (mean-SD; $n=2-3$ ). (C) Absolute number of total BM cells and (E) relative numbers of BM stromal cell subsets and BM-MSCs in ARE-Del mice and WT controls (mean-SEM; $n=5$ ). (D) Gating strategy for murine BM MSCs (F) CXCL12 and SCF expression by sorted BM-MSC, normalized to Cyclophilin (mean-SEM; $n=5$ ). (G) Percentage of BM-MSCs in total BM cells of ARE-Del mice and WT controls between 3-30 weeks of age (mean-SEM; $n=3-5)$. $* P<0.05$; $* * * P<0.001$ unpaired $t$-test. BM, bone marrow; WT, wild type; SEM, standard error of the mean; ARE, AU-rich elements. 
factors produced by MSC-y that inhibit myeloid skewing. The difference found on myeloid progenitors assessed by flow cytometry versus colony assays (Fig. 1E, D) probably relates to the fact that only a subset of CD34+ cells is able to give rise to colonies. It is most likely that the small increase in HSPCs remaining at the end of the coculture is of a subset that does not have significant colony-forming potential.

A recent study by Yang et al. claimed that IFN- $\gamma$ induces senescence-like characteristics in murine MSC, which could explain our findings that MSC maintenance is impaired upon exposure to IFN- $\gamma$ [43]. In contrast, Chinnadurai et al., showed that prelicensing with IFN- $\gamma$ can correct some of the senescence-associated characteristics of cultured human MSC [44]. Thus, the exact mechanism by which IFN- $\gamma$ influences BM-MSCs is still under debate and arguably depends greatly on the duration of the exposure. Prelicensing with a short exposure of IFN- $\gamma$ enhances immunomodulatory capacities of cultured human MSC, and may therefore be an attractive strategy for clinical application in inflammatory conditions (reviewed in Ref. [11]), especially because it has recently been suggested that the clinical risk of infusing $\mathrm{MSC} \gamma$ is not greater than the risk of infusing nontreated MSCs [45]. It has also been reported that IFN- $\gamma$ exposure of MSCs can mediate a shift from adipogenesis toward osteoblastogenesis both in vitro and in vivo [30], which may be beneficial for clinical application of cultured human MSC in bone degenerative disorder. However, our data argue against using longer IFN- $\gamma$ exposure of cultured MSC destined for clinical application, as it negatively affects their maintenance. In addition, our data do not support a positive role for IFN- $\gamma$ in the hematopoietic support of BM-MSC, and rather argue against using IFN- $\gamma$-stimulated MSCs for hematopoietic support in a clinical setting. A definitive answer on this matter may be provided by experiments, where BM-MSCs cultured in the presence or absence of IFN- $\gamma$ are infused during a murine BM transplantation, and engraftment of HSCs and hematopoietic reconstitution are subsequently evaluated.

In addition to our findings that IFN- $\gamma$ does not boost the hematopoietic support function of in vitro-expanded MSCs, we identified a negative role for IFN- $\gamma$ on MSC in vivo in the $\mathrm{BM}$ niche. Chronic exposure to IFN- $\gamma$ in mice resulted in strongly reduced numbers of BM-MSCs, which correlated with a concomitant loss of quiescent HSC. Although HSCs and downstream progenitors can also directly respond to high levels of IFN- $\gamma$ (reviewed in Ref. [23]), we found no upregulation of the interferon-sensitive molecule Sca-1 on HSPCs in ARE-Del mice (Fig. 3a; Supplementary Fig. S8), in contrast to other models, in which higher levels of IFN- $\gamma$ are produced [22]. Therefore, it is highly unlikely that the observed HSC phenotype in the ARE-Del mice is due to a direct effect of IFN- $\gamma$. In support of this, also acute production of IFN- $\gamma$ by adoptively transferred activated $\mathrm{T}$ cells, which does induce Sca-1 upregulation on most BM cells, is not sufficient to affect HSC numbers [34]. Moreover, we previously showed that direct IFN- $\gamma$ exposure itself does not affect HSC quiescence [22]. Altogether, these arguments thus imply that the observed loss in HSC quiescence in ARE-Del mice is due to the detrimental effect of IFN- $\gamma$ on the HSC niche. This is supported by recent work from O'Hagan-Wong et al., in which aging and DNA damage in niche cells is coupled to loss of HSC quiescence [46]. The concept that stress-induced changes in stromal cells can affect the hematopoietic process has also been recently proposed by Zambetti et al., who found that perturbation of MSCs in a preleukemic mouse model induces genotoxic stress in HSPCs and can predict disease evolution in human preleukemia disorders [47]. Another form of stress-induced changes in stromal cells has been reported by Abbuehl et al., who show that MSCs are severely impaired following upon preconditioning irradiation for HSCT, which limits the reconstitution of donor-derived HSC [48]. In addition, Schurch et al. have reported that IL-6 production by BM stromal cells upon inflammation can induce a myeloid bias in hematopoiesis and thus increase myeloid output of the BM [34]. We previously showed that chronic IFN- $\gamma$ production contributes to the development of acquired aplastic anemia (AAA) and we suggested that nonhematopoietic cells could be involved in driving the loss of HSC [49]. This is corroborated by our current findings that IFN- $\gamma$-mediated depletion of BM-MSCs in IFN- $\gamma$ AREDel mice coincides with reduced quiescence of LT-HSC, increased differentiation toward more committed progenitor cells, and exhaustion of the LT-HSC pool upon aging.

Our definition of murine BM-MSCs as CD45 Ter119 $\mathrm{CD} 31^{-} \mathrm{PDGFR} \alpha^{+} \mathrm{CD} 51^{+}$cells encompasses a population with high expression of CXCL12. Although we did not observe reduced CXCL12 expression by human MSC- $\gamma$ nor BM-MSCs from ARE-Del mice, the decrease in total BMMSC numbers leads to a reduction in CXCL12-producing HSC niches. When measuring the total amount of CXCL12 protein in BM supernatant, we could not find a significant difference between WT and ARE-Del mice (data not shown), though this could be due to the fact that the number of CXCL12-producing cells in the BM is much higher than the number of HSC-supporting niches, as endothelial cells and stromal cells other than MSCs can also produce this chemokine. Studies by Ding and Morrison and Tzeng et al. showed that conditional deletion of CXCL12 only from Prx $1^{+}$ and $\mathrm{Osx}^{+}$stromal precursor cells results in loss of HSC quiescence $[41,50]$, which is consistent with our findings. Additionally, loss of HSC quiescence upon CXCL12 deletion leads to an expansion of the hematopoietic progenitor pool $[41,50]$. Thus, a reduction in the number of CXCL12 ${ }^{+}$niches in the BM could explain the loss of quiescent LT-HSC and increased differentiation to progenitors in ARE-Del mice. Our results are therefore congruent with the findings that the production of CXCL12 by BM-MSCs in the vascular BM niche is essential for maintaining the balance between quiescence and proliferation in HSCs [3].

By combining results on human BM-MSCs as well as mouse BM-MSCs, in vivo and in vitro, we provide clinical relevance as well as biological significance. An important implication of our results is that prestimulation of BMMSCs with IFN- $\gamma$ for clinical purposes is not favorable for hematopoietic support, thus limiting the benefits of using IFN- $\gamma$-stimulated BM-MSCs in BM transplantation settings. The findings of this study may also contribute to the understanding of the underlying causes of AAA. Several studies showed defective proliferation and differentiation of BMMSCs from AAA patients [51-53], which are known to have increased levels of circulating IFN- $\gamma$ [54]. One study demonstrated reduced outgrowth of $\mathrm{CD} 34^{+}$HSPCs when cocultured with AAA BM-MSCs compared with healthy stroma, 


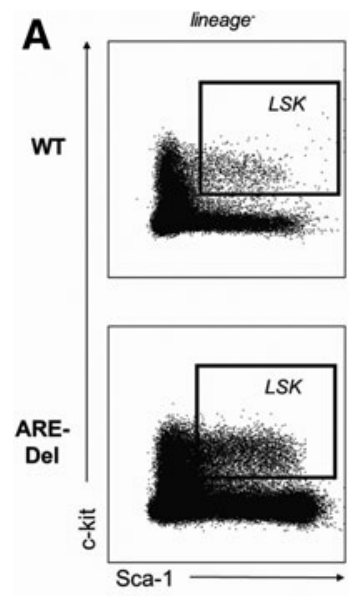

D
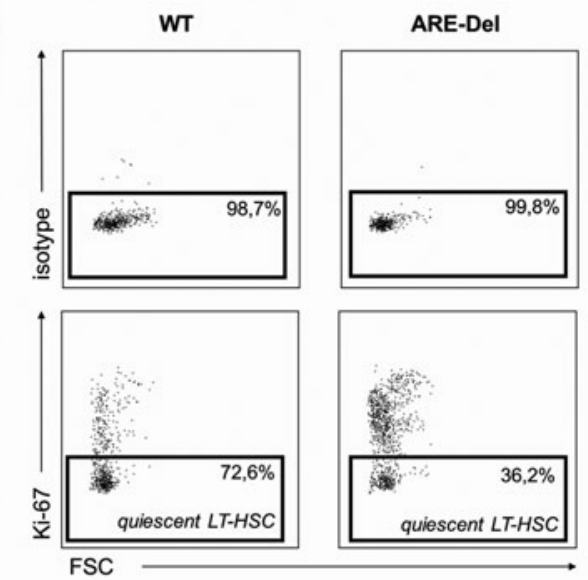

$\mathbf{F}$

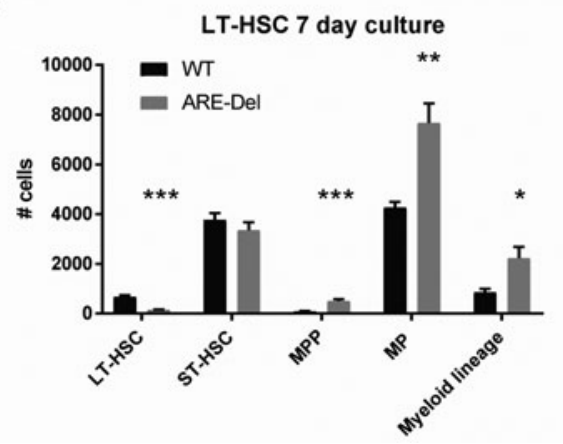

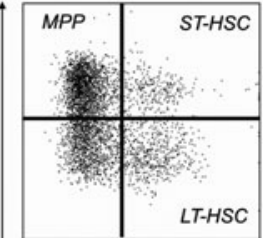

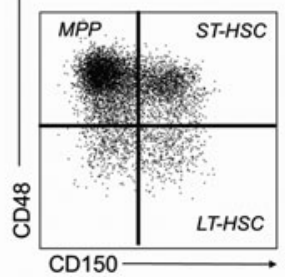

ARE-Del

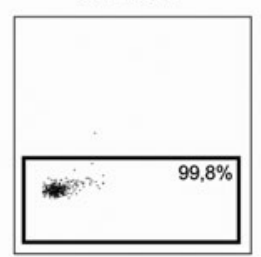

G

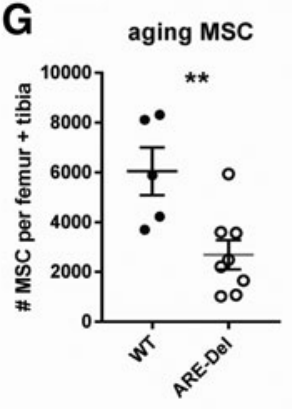

$\mathbf{E}$
B
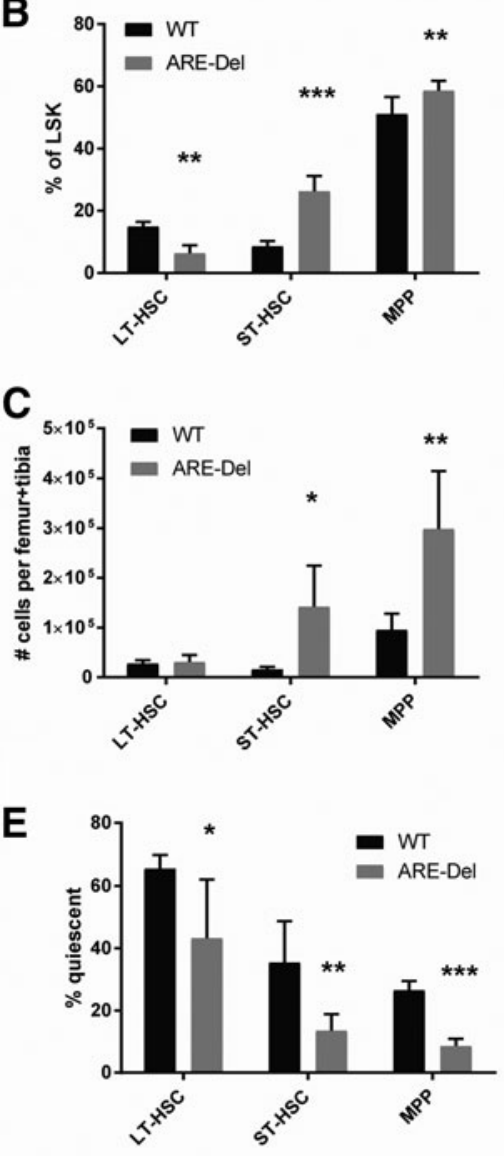

H

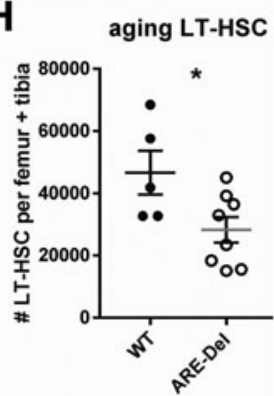

FIG. 3. $\mathrm{HS}(\mathrm{P}) \mathrm{C}$ numbers and quiescence in ARE-Del mice. (A) Gating strategy for SLAM HSC. (B) Percentage of LSK and (C) absolute number of $\mathrm{HS}(\mathrm{P}) \mathrm{C}$ in ARE-Del mice and WT controls (mean-SEM; $n=5)$. (D) Flow cytometric analysis and (E) quantification of Ki-67-negative $\mathrm{HS}(\mathrm{P}) \mathrm{C}$ in ARE-Del mice and WT controls (mean-SEM; $n=5)(\mathbf{F})$ LT-HSC were sorted and cultured for 7 days in X-vivo medium with TPO, SCF, IL3, IL-6, and Flt3-L. After 7 days, flow cytometric analysis was performed to identify phenotypic HS(P)Cs (meanSEM; $n=7-8$ ). (G) Absolute numbers of BM-MSCs and (H) LT-HSC in 1-year-old ARE-Del mice and WT controls. $* P<0.05 ; \quad * * P<0.01$, $* * * P<0.001$ unpaired $t$-test. HSC, hematopoietic stem cells; LT-HSC, long-term HSC; TPO, thrombopoietin; LSK, Lineage ${ }^{-}$Sca-1 ${ }^{+}$c-kit $^{+}$. consistent with our findings on reduced hematopoietic support by culture-expanded BM-MSCs pretreated with IFN- $\gamma$ [51]. In contrast, others have reported no functional defects in MSCs derived from AAA patients [55]. Thus, there might be a role for IFN- $\gamma$-stimulated BM-MSCs in the pathology of AAA, but the underlying mechanism and the extent of this effect should be addressed by further research. In conclusion, IFN- $\gamma$ does not increase hematopoietic support of BM-MSC, but rather has negative effect on their maintenance, which is correlated with decreased support for quiescent HSCs in vivo. This knowledge provides an argument against the use of IFN- $\gamma$ priming of MSCs for clinical therapies that aim to enhance hematopoietic engraftment. In addition, our work offers valuable insight into the impact of an inflammatory environment on the BM as a supporting organ for hematopoiesis.

\section{Acknowledgments}

The authors thank Mark Hoogenboezem, Simon Tol, and Erik Mul for technical assistance and the staff of the animal facility of the NKI for excellent animal care. M.G., J.D.v.B., S.H., and M.A.N. were financially supported by Sanquin Research (PPOC13-030P grant). S.G. was financially supported by a Fellowship obtained by M.A.N. from the Landsteiner Foundation for Blood Transfusion Research, grant no. \#1014 (www.lsbr.nl). A.S.C. and C.V. were financially supported by a Fellowship obtained by C.V. from the Landsteiner Foundation for Blood Transfusion Research, grant no. \#1101. This research was supported, in part, by the Intramural Research Program of the NIH, National Cancer Institute. 


\section{Author Disclosure Statement}

No competing financial interests exist.

\section{References}

1. Morrison SJ and DT Scadden. (2014). The bone marrow niche for haematopoietic stem cells. Nature 505:327-334.

2. Ding L, TL Saunders, G Enikolopov and SJ Morrison. (2012). Endothelial and perivascular cells maintain haematopoietic stem cells. Nature 481:457-462.

3. Greenbaum A, YM Hsu, RB Day, LG Schuettpelz, MJ Christopher, JN Borgerding, T Nagasawa and DC Link. (2013). CXCL12 in early mesenchymal progenitors is required for haematopoietic stem-cell maintenance. Nature 495:227-230.

4. Beyer Nardi N and L da Silva Meirelles. (2006). Mesenchymal stem cells: isolation, in vitro expansion and characterization. Handb Exp Pharmacol 174:249-282.

5. Pittenger MF, AM Mackay, SC Beck, RK Jaiswal, R Douglas, JD Mosca, MA Moorman, DW Simonetti, S Craig and DR Marshak. (1999). Multilineage potential of adult human mesenchymal stem cells. Science 284:143-147.

6. Majumdar MK, MA Thiede, SE Haynesworth, SP Bruder and SL Gerson. (2000). Human marrow-derived mesenchymal stem cells (MSCs) express hematopoietic cytokines and support long-term hematopoiesis when differentiated toward stromal and osteogenic lineages. J Hematother Stem Cell Res 9:841-848.

7. Horwitz EM, DJ Prockop, LA Fitzpatrick, WW Koo, PL Gordon, M Neel, M Sussman, P Orchard, JC Marx, RE Pyeritz and MK Brenner. (1999). Transplantability and therapeutic effects of bone marrow-derived mesenchymal cells in children with osteogenesis imperfecta. Nat Med 5:309-313.

8. Krampera M. (2011). Mesenchymal stromal cell "licensing": a multistep process. Leukemia 25:1408-1414.

9. Le Blanc K, F Frassoni, L Ball, F Locatelli, H Roelofs, I Lewis, E Lanino, B Sundberg, ME Bernardo, et al.; Developmental Committee of the European Group for Blood and Marrow Transplantation. (2008). Mesenchymal stem cells for treatment of steroid-resistant, severe, acute graft-versushost disease: a phase II study. Lancet 371:1579-1586.

10. Le Blanc K, H Samuelsson, B Gustafsson, M Remberger, B Sundberg, J Arvidson, P Ljungman, H Lonnies, S Nava and O Ringden. (2007). Transplantation of mesenchymal stem cells to enhance engraftment of hematopoietic stem cells. Leukemia 21:1733-1738.

11. Sivanathan KN, S Gronthos, D Rojas-Canales, B Thierry and PT Coates. (2014). Interferon-gamma modification of mesenchymal stem cells: implications of autologous and allogeneic mesenchymal stem cell therapy in allotransplantation. Stem Cell Rev 10:351-375.

12. Tobin LM, ME Healy, K English and BP Mahon. (2013). Human mesenchymal stem cells suppress donor CD4(+) T cell proliferation and reduce pathology in a humanized mouse model of acute graft-versus-host disease. Clin Exp Immunol 172:333-348.

13. Polchert D, J Sobinsky, G Douglas, M Kidd, A Moadsiri, E Reina, K Genrich, S Mehrotra, S Setty, B Smith and A Bartholomew. (2008). IFN-gamma activation of mesenchymal stem cells for treatment and prevention of graft versus host disease. Eur J Immunol 38:1745-1755.

14. Liu Y, L Wang, T Kikuiri, K Akiyama, C Chen, X Xu, R Yang, W Chen, S Wang and S Shi. (2011). Mesenchymal stem cellbased tissue regeneration is governed by recipient $\mathrm{T}$ lymphocytes via IFN-gamma and TNF-alpha. Nat Med 17:1594-1601.
15. Duijvestein M, ME Wildenberg, MM Welling, S Hennink, I Molendijk, VL van Zuylen, T Bosse, AC Vos, ES de JongeMuller, et al. (2011). Pretreatment with interferon-gamma enhances the therapeutic activity of mesenchymal stromal cells in animal models of colitis. Stem Cells 29:1549-1558.

16. Cornelissen AS, MW Maijenburg, MA Nolte and C Voermans. (2015). Organ-specific migration of mesenchymal stromal cells: who, when, where and why? Immunol Lett 168:159-169.

17. Maijenburg MW, CE van der Schoot and C Voermans. (2012). Mesenchymal stromal cell migration: possibilities to improve cellular therapy. Stem Cells Dev 21:19-29.

18. Chinnadurai R, IB Copland, MA Garcia, CT Petersen, CN Lewis, EK Waller, AD Kirk and J Galipeau. (2016). Cryopreserved mesenchymal stromal cells are susceptible to T-cell mediated apoptosis which is partly rescued by IFNgamma licensing. Stem Cells 34:2429-2442.

19. English K, BP Mahon and KJ Wood. (2014). Mesenchymal stromal cells; role in tissue repair, drug discovery and immune modulation. Curr Drug Deliv 11:561-571.

20. Yang L, I Dybedal, D Bryder, L Nilsson, E Sitnicka, Y Sasaki and SE Jacobsen. (2005). IFN-gamma negatively modulates self-renewal of repopulating human hemopoietic stem cells. J Immunol 174:752-757.

21. Matatall KA, CC Shen, GA Challen and KY King. (2014). Type II interferon promotes differentiation of myeloidbiased hematopoietic stem cells. Stem Cells 32:3023-3030.

22. de Bruin AM, O Demirel, B Hooibrink, CH Brandts and MA Nolte. (2013). Interferon-gamma impairs proliferation of hematopoietic stem cells in mice. Blood 121:3578-3585.

23. de Bruin AM, C Voermans and MA Nolte. (2014). Impact of interferon-gamma on hematopoiesis. Blood 124:2479-2486.

24. Paciejewska MM, MW Maijenburg, C Gilissen, M Kleijer, K Vermeul, K Weijer, JA Veltman, M von Lindern, CE van der Schoot and C Voermans. (2016). Different balance of Wnt signaling in adult and fetal bone marrow-derived mesenchymal stromal cells. Stem Cells Dev 25:934-947.

25. Klamer SE, CG Kuijk, PL Hordijk, CE van der Schoot, M von Lindern, PB van Hennik and C Voermans. (2013). BIGH3 modulates adhesion and migration of hematopoietic stem and progenitor cells. Cell Adh Migr 7:434-449.

26. Hodge DL, C Berthet, V Coppola, W Kastenmuller, MD Buschman, PM Schaughency, H Shirota, AJ Scarzello, JJ Subleski, et al. (2014). IFN-gamma AU-rich element removal promotes chronic IFN-gamma expression and autoimmunity in mice. J Autoimmun 53:33-45.

27. Mignone JL, V Kukekov, AS Chiang, D Steindler and G Enikolopov. (2004). Neural stem and progenitor cells in nestin-GFP transgenic mice. J Comp Neurol 469:311-324.

28. Le Blanc K, C Tammik, K Rosendahl, E Zetterberg and O Ringden. (2003). HLA expression and immunologic properties of differentiated and undifferentiated mesenchymal stem cells. Exp Hematol 31:890-896.

29. Dominici M, K Le Blanc, I Mueller, I Slaper-Cortenbach, F Marini, D Krause, R Deans, A Keating, D Prockop and E Horwitz. (2006). Minimal criteria for defining multipotent mesenchymal stromal cells. The International Society for Cellular Therapy position statement. Cytotherapy 8:315-317.

30. Vidal C, S Bermeo, W Li, D Huang, R Kremer and G Duque. (2012). Interferon gamma inhibits adipogenesis in vitro and prevents marrow fat infiltration in oophorectomized mice. Stem Cells 30:1042-1048.

31. Duque G, DC Huang, M Macoritto, D Rivas, XF Yang, LG Ste-Marie and R Kremer. (2009). Autocrine regulation of 
interferon gamma in mesenchymal stem cells plays a role in early osteoblastogenesis. Stem Cells 27:550-558.

32. Croitoru-Lamoury J, FM Lamoury, M Caristo, K Suzuki, D Walker, O Takikawa, R Taylor and BJ Brew. (2011). Interferon-gamma regulates the proliferation and differentiation of mesenchymal stem cells via activation of indoleamine 2,3 dioxygenase (IDO). PLoS One 6:e14698.

33. Ishihara $\mathrm{K}$ and $\mathrm{T}$ Hirano. (2002). IL-6 in autoimmune disease and chronic inflammatory proliferative disease. Cytokine Growth Factor Rev 13:357-368.

34. Schurch CM, C Riether and AF Ochsenbein. (2014). Cytotoxic $\mathrm{CD} 8+\mathrm{T}$ cells stimulate hematopoietic progenitors by promoting cytokine release from bone marrow mesenchymal stromal cells. Cell Stem Cell 14:460-472.

35. Kerst JM, IC Slaper-Cortenbach, CE van der Schoot, B Hooibrink, AE von dem Borne and RH van Oers. (1993). Interleukin-6 is a survival factor for committed myeloid progenitor cells. Exp Hematol 21:1550-1557.

36. Jing D, AV Fonseca, N Alakel, FA Fierro, K Muller, M Bornhauser, G Ehninger, D Corbeil and R Ordemann. (2010). Hematopoietic stem cells in co-culture with mesenchymal stromal cells-modeling the niche compartments in vitro. Haematologica 95:542-550.

37. Oubari F, N Amirizade, H Mohammadpour, M Nakhlestani and MN Zarif. (2015). The important role of FLT3-L in ex vivo expansion of hematopoietic stem cells following coculture with mesenchymal stem cells. Cell J 17:201-210.

38. Pinho S, J Lacombe, M Hanoun, T Mizoguchi, I Bruns, Y Kunisaki and PS Frenette. (2013). PDGFRalpha and CD51 mark human nestin+ sphere-forming mesenchymal stem cells capable of hematopoietic progenitor cell expansion. J Exp Med 210:1351-1367.

39. Mendez-Ferrer S, TV Michurina, F Ferraro, AR Mazloom, BD Macarthur, SA Lira, DT Scadden, A Ma'ayan, GN Enikolopov and PS Frenette. (2010). Mesenchymal and haematopoietic stem cells form a unique bone marrow niche. Nature 466:829-834.

40. Oikonomopoulos A, WK van Deen, AR Manansala, PN Lacey, TA Tomakili, A Ziman and DW Hommes. (2015). Optimization of human mesenchymal stem cell manufacturing: the effects of animal/xeno-free media. Sci Rep 5:16570.

41. Tzeng YS, H Li, YL Kang, WC Chen, WC Cheng and DM Lai. (2011). Loss of Cxcl12/Sdf-1 in adult mice decreases the quiescent state of hematopoietic stem/progenitor cells and alters the pattern of hematopoietic regeneration after myelosuppression. Blood 117:429-439.

42. Kiel MJ, OH Yilmaz, T Iwashita, OH Yilmaz, C Terhorst and SJ Morrison. (2005). SLAM family receptors distinguish hematopoietic stem and progenitor cells and reveal endothelial niches for stem cells. Cell 121:1109-1121.

43. Yang ZX, GX Mao, J Zhang, XL Wen, BB Jia, YZ Bao, XL Lv, YZ Wang and GF Wang. (2017). IFN-gamma induces senescence-like characteristics in mouse bone marrow mesenchymal stem cells. Adv Clin Exp Med 26:201-206.

44. Chinnadurai R, D Rajan, S Ng, K McCullough, D Arafat, EK Waller, LJ Anderson, G Gibson and J Galipeau. (2017). Immune dysfunctionality of replicative senescent mesenchymal stromal cells is corrected by IFNgamma priming. Blood Adv 1:628-643.

45. Guess AJ, B Daneault, R Wang, H Bradbury, KMD La Perle, J Fitch, SL Hedrick, E Hamelberg, C Astbury, et al. (2017). Safety profile of good manufacturing practice manufactured interferon gamma-primed mesenchymal stem/stromal cells for clinical trials. Stem Cells Transl Med 6:1868-1879.

46. O’Hagan-Wong K, S Nadeau, A Carrier-Leclerc, F Apablaza, R Hamdy, D Shum-Tim, F Rodier and I Colmegna. (2016). Increased IL-6 secretion by aged human mesenchymal stromal cells disrupts hematopoietic stem and progenitor cells' homeostasis. Oncotarget 7:13285-13296.

47. Zambetti NA, Z Ping, S Chen, KJ Kenswil, MA Mylona, MA Sanders, RM Hoogenboezem, EM Bindels, MN Adisty, et al. (2016). Mesenchymal inflammation drives genotoxic stress in hematopoietic stem cells and predicts disease evolution in human pre-leukemia. Cell Stem Cell 19:613-627.

48. Abbuehl JP, Z Tatarova, W Held and J Huelsken. (2017). Long-term engraftment of primary bone marrow stromal cells repairs niche damage and improves hematopoietic stem cell transplantation. Cell Stem Cell 21:241-255 e6.

49. Lin FC, M Karwan, B Saleh, DL Hodge, T Chan, KC Boelte, JR Keller and HA Young. (2014). IFN-gamma causes aplastic anemia by altering hematopoietic stem/ progenitor cell composition and disrupting lineage differentiation. Blood 124:3699-3708.

50. Ding L and SJ Morrison. (2013). Haematopoietic stem cells and early lymphoid progenitors occupy distinct bone marrow niches. Nature 495:231-235.

51. Hamzic E, K Whiting, E Gordon Smith and R Pettengell. (2015). Characterization of bone marrow mesenchymal stromal cells in aplastic anaemia. Br J Haematol 169:804813.

52. Chao YH, CT Peng, HJ Harn, CK Chan and KH Wu. (2010). Poor potential of proliferation and differentiation in bone marrow mesenchymal stem cells derived from children with severe aplastic anemia. Ann Hematol 89:715-723.

53. Li J, S Yang, S Lu, H Zhao, J Feng, W Li, F Ma, Q Ren, B Liu, et al. (2012). Differential gene expression profile associated with the abnormality of bone marrow mesenchymal stem cells in aplastic anemia. PLoS One 7:e47764.

54. Zoumbos NC, P Gascon, JY Djeu and NS Young. (1985). Interferon is a mediator of hematopoietic suppression in aplastic anemia in vitro and possibly in vivo. Proc Natl Acad Sci U S A 82:188-192.

55. Michelozzi IM, A Pievani, F Pagni, L Antolini, M Verna, $P$ Corti, A Rovelli, M Riminucci, F Dazzi, A Biondi and M Serafini. (2016). Human aplastic anaemia-derived mesenchymal stromal cells form functional haematopoietic stem cell niche in vivo. Br J Haematol 179:669-673.

Address correspondence to: Dr. Martijn A. Nolte Department of Hematopoiesis, Sanquin Research and Landsteiner Laboratory AMC/UvA

Plesmanlaan 125

1066CX Amsterdam Netherlands

E-mail: m.nolte@sanquin.nl

Received for publication September 21, 2017 Accepted after revision March 12, 2018 Prepublished on Liebert Instant Online March 12, 2018 